\title{
EDITOR'S
}

\section{Bladder Cancer: What we can do against this powerful enemy?}

The March-April 2014 issue of the International Braz J Urol presents original contributions with a lot papers in different fields. The papers come from many different countries such as Brazil, USA, China, Italy, Japan, Chile and Turkey, and as usual the editor's comment highlights some papers. In this number we can observe some papers about bladder cancer. The bladder cancer represents a clinical challenge for the urologist and in this number we had papers about the clinical diagnosis, prognostic markers, radiation therapy and the natural history of the high-grade T1 bladder cancer. The high-grade T1 bladder cancer is a very dangerous disease and the time of indication of radical cystectomy is very important in this treatment. We summarize the papers about bladder cancer below.

Doctor Canter and collegues, from the Departments of Urology, from the university from Pennsylvania, USA performed on page 172 an interesting study Using two non-muscle invasive bladder cancer (NMIBC) databases, the authors re-examined the rate of progression of HG T1 bladder cancer in your bladder cancer populations. A total of 222 patients were identified and the authors found that the risk of progression at approximately four years was only $8.6 \%$. This study could potentially serve as a starting point in re-examining the treatment algorithm for patients with HG T1 bladder cancer

Doctor Zhao and collegues from the Qingdao University, Qungdao, China performed on page 179 a elegant study about prognostic markers in bladder cancer. The goal of this study was to utilize long-term patient follow-up to determine whether epithelial-to-mesenchymal transition (EMT)-related markers can predict bladder cancer patient survival and progression of disease. The authors make the analysis of 121 bladder cancer patients and revealed that the frequency of E-cadherin expression was 


\section{EDITOR'S}

59.5\% (72/121), Twist was 54.5\% (66/121), and Vimentin was 24.8\% (30/121). In this analysis, grade and Vimentin expression were found to be significant prognostic factors in predicting progression-free survival in bladder cancer.

Doctor Freilich and collegues from the Moffitt Cancer Center, Florida, USA performed on page 190 a study about the evaluation of Lipiodol as a liquid, radio-opaque fiducial marker for image-guided radiation therapy (IGRT) for bladder cancer. They studied 5 clinical T2a-T3b N0 M0 stage II-III bladder cancer patients were treated with maximal transurethral resection of a bladder tumor (TURBT) and image-guided radiation therapy (IGRT) to 64.8 Gy in 36 fractions \pm concurrent weekly cisplatin-based or gemcitabine chemotherapy and concluded that Lipiodol constitutes a safe and effective fiducial marker that an urologist can use to demarcate a tumor bed immediately following maximal TURBT. Lipiodol decreases inter-observer variability in the definition of the extent and location of a tumor bed on a treatment planning CT scan for a radiation boost.

Doctor Dobbs and collegues from the Emory University School of Medicine, Atlanta, USA performed on page 198 a interesting clinical study about the incidence of lower urinary tract symptoms (LUTS) as the sole presenting symptom for bladder cancer. The authors evaluated the prevalence and clinical characteristics of newly diagnosed bladder cancer patients who presented with LUTS in the absence of gross or microscopic hematuria. They studied 340 patients and observed that $4.1 \%$ of bladder cancer patients in our series presented solely with LUTS. In this cohort a small percentage of patients with newly diagnosed bladder cancer present with LUTS without gross or microscopic 


\section{EDITOR'S

hematuria. Despite a higher incidence of CIS com- pared to patients with other presenting symptoms, the majority of patients with LUTS presented with Ta lesions. This study suggests that urologists should have a low threshold for evaluating patients with unexplained LUTS for underlying bladder cancer.

\section{Luciano A. Favorito, MD, PhD}

Associated Professor of Urogenital Research Unit State University from Rio de Janeiro - UERJ 